\title{
Theory of reduced singlet pairing without the underlying state of charge stripes in the high-temperature superconductor $\mathrm{YBa}_{2} \mathrm{Cu}_{3} \mathrm{O}_{6.45}$
}

\author{
Hiroyuki Yamase \\ Max-Planck-Institute for Solid State Research, \\ Heisenbergstrasse 1, D-70569 Stuttgart, Germany
}

(Dated: October 26, 2018)

\begin{abstract}
Recently, a strongly enhanced $x y$ anisotropy of magnetic excitations was observed in $\mathrm{YBa}_{2} \mathrm{Cu}_{3} \mathrm{O}_{y}$ $\left(\mathrm{YBCO}_{y}\right)$ with $y=6.45$ and $T_{c}=35 \mathrm{~K}$ [Science 319, 597 (2008)]. Unlike the observation in $\mathrm{YBCO}_{6.6}$ and $\mathrm{YBCO}_{6.85}$, the anisotropy grows to be pronounced at lower temperature and at lower energy, and is not suppressed by the onset of superconductivity. We propose that the effect of singlet pairing is substantially reduced in $\mathrm{YBCO}_{6.45}$. This reduction concomitantly enhances an order competing with singlet pairing, a strong tendency of the so-called $d$-wave Pomeranchuk instability, leading to the magnetic excitations observed experimentally.

PACS numbers: 74.25.Ha, 74.72.Bk, 74.20.Mn, 71.10.Fd
\end{abstract}


The spin-charge stripe order was widely discussed in high- $T_{c}$ cuprates. [1] In this scenario, the two-dimensional $\mathrm{CuO}_{2}$ plane is assumed to have the instability of one-dimensional charge order, the so-called stripe pattern, in the antiferromagnetic (AF) background with a $\pi$ phase shift across the charge stripe. However, the charge order signal was observed only for limited materials with specific hole doping rates and had a broad spectrum with very weak intensity, [2, 3, 4, 5] implying that the charge order is usually not well developed. Kivelson, Fradkin, and Emery introduced a new concept of electronic nematic order, [6] which was envisaged as the melting of charge stripe order, [7] where the translational symmetry is recovered but the orientational symmetry is still broken.

On the other hand, an alternative root to yield electronic nematic order was found in minimal models of high- $T_{c}$ cuprates such as the $t-J[8,9$, 10] and Hubbard [11, 12, 13, 14, 15] models. These models show a tendency toward $d$-wave type Fermi surface deformations ( $d$ FSD), the so-called $d$-wave Pomeranchuk instability. The Fermi surface (FS) expands along the $k_{x}$ direction and shrinks along the $k_{y}$ direction, or vice versa. The $d$ FSD state has the same symmetry as the electronic nematic state. But the physical origin is different from the stripe physics, [6] since the $d \mathrm{FSD}$ is generated by forward scattering processes of quasiparticles, not by fluctuating charge stripes.

The $d$ FSD competes with superconductivity. [16] In the slave-boson mean-field[8] and variational Monte Carlo[10] analyses of the $t$ - $J$ model, superconductivity becomes dominant. Yet the system still has sizable $d$ FSD correlations, [17] producing a giant response to a small external anisotropy, e.g., due to a lattice structure and anisotropic strain. [8, 10] This theoretical insight yields a promising scenario[18] to understand the pronounced anisotropy of magnetic excitations observed in untwinned $\mathrm{YBa}_{2} \mathrm{Cu}_{3} \mathrm{O}_{y}\left(\mathrm{YBCO}_{y}\right)$ with $y=6.5$, [19] $6.6,[20,21]$ and $6.85,[20]$ where the lattice yields a small $x y$ anisotropy.

Quite recently, a very strong anisotropy was observed in magnetic excitations in untwinned $\mathrm{YBCO}_{6.45} \cdot[22]$ The observed spectra were qualitatively different from typical observations in Y-based cuprates. (i) The anisotropy starts to increase below $\sim 150 \mathrm{~K}$ and saturates below $\sim 50 \mathrm{~K}$, in contrast to the case of $\mathrm{YBCO}_{6.6}[20,21]$ where the anisotropy is most pronounced at relatively high temperature and is reduced at low temperature. (ii) The magnetic excitation spectra are hardly affected by the onset of superconductivity and in fact a gap feature is not found in the magnetic excitations. This is sharply different from the well-known observation in Y-based cuprates, where a broad spectrum around $\mathbf{q}=(\pi, \pi)$ 
becomes very sharp and is strongly enhanced below the onset temperature of superconductivity, leading to a resonance at relatively high energy, followed by suppression of low energy spectral weight. [23, 24] These peculiar magnetic excitations in $\mathrm{YBCO}_{6.45}$ are not captured even qualitatively by simple application of the theory in Ref. 18, implying failure of the standard RVB mean-field theory in a strongly underdoped region.

Why do the qualitative features of magnetic excitations change so drastic in $\mathrm{YBCO}_{6.45}$ ? Since $\mathrm{YBCO}_{6.45}$ lies closer to the AF instability, strong AF fluctuations might play an important role. However, even for the lower hole-doped system $\mathrm{YBCO}_{6.353}$, and thus much closer to the AF instability, the experimental data[25] showed that magnetic correlation length is still short ranged. In this paper, we show an integral role of $d$ FSD correlations, which are concomitantly enhanced by the suppression of singlet pairing, leading to results very similar to the experimental observation. [22]

We analyze the bilayer $t$ - $J$ model on a square lattice, a minimal model for Y-based cuprates, [26]

$$
H=-\sum_{\mathbf{r}, \mathbf{r}^{\prime}, \sigma} t_{\tau} \tilde{c}_{\mathbf{r} \sigma}^{\dagger} \tilde{C}_{\mathbf{r}^{\prime} \sigma}+\sum_{\left\langle\mathbf{r}, \mathbf{r}^{\prime}\right\rangle} J_{\tau} \mathbf{S}_{\mathbf{r}} \cdot \mathbf{S}_{\mathbf{r}^{\prime}}
$$

defined in the Fock space with no doubly occupied sites. The operator $\tilde{c}_{\mathbf{r} \sigma}^{\dagger}\left(\tilde{c}_{\mathbf{r} \sigma}\right)$ creates (annihilates) an electron with spin $\sigma$ on site $\mathbf{r}$, and $\mathbf{S}_{\mathbf{r}}$ is the spin operator. $J_{\tau}(>0)$ is a superexchange coupling between the nearest neighbor sites along each direction, $\tau=x, y, z$. We take into account hopping amplitudes $t_{\tau}$ between $\mathbf{r}$ and $\mathbf{r}^{\prime}$ up to third-nearest neighbors, and the direction of $\mathbf{r}^{\prime}-\mathbf{r}$ is represented by $\tau$. The Hamiltonian (1) is analyzed in the standard slave-boson method in the $t$ - $J$ model by introducing the so-called resonating-valence-bond (RVB) mean fields. However, in contrast to the standard RVB theory, we assume the mean field of singlet pairing $\Delta$ to be zero, in order to mimic possibly substantial suppression of singlet pairing in $\mathrm{YBCO}_{6.45}$ as we will discuss later in detail. The formalism and the band parameters are otherwise the same as those in Ref. 18, where we successfully discussed the pronounced anisotropy of magnetic excitations in $\mathrm{YBCO}_{6.85}[20]$ and $\mathrm{YBCO}_{6.6} \cdot[20,21] \mathrm{We}$ introduce 5\% xy anisotropy into the hopping integrals $t_{\tau}$ and $10 \%$ into the superexchange coupling $J_{\tau}$, twice as large, as imposed by the superexchange mechanism. The anisotropy then can be parameterized by a single parameter $\alpha=-0.05$. The minus sign indicates that the band parameters are more enhanced along the $y$ direction, parallel to the direction of the $\mathrm{CuO}$ chains, which indeed reproduces [18, 27, 28] qualitatively the same anisotropic dis- 


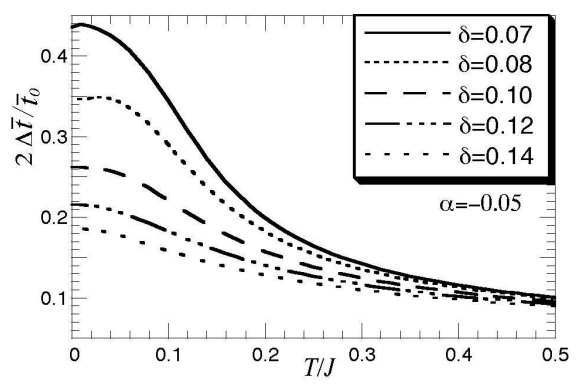

FIG. 1: $T$ dependence of the anisotropy of renormalized band, $2 \Delta \bar{t} / \Delta \bar{t}_{0}$, for several choices of $\delta$ for $\alpha=-0.05$.

tribution of magnetic excitations as observed previously. [20, 21] Interestingly the negative sign of $\alpha$ is also implied in a spin spiral state to understand the anisotropy of magnetic excitations. [29]

The most important quantity is the renormalized in-plane nearest-neighbor hopping $\bar{t}_{\tau}$, which is given by $\bar{t}_{\tau}=t_{\tau} \delta+\frac{3}{8} J_{\tau} \chi_{\tau}$ with $\tau=x, y ; \delta$ is the hole density and $\chi_{\tau}$ is the RVB bond order along the $\tau$ direction. The order parameter of the $d$ FSD is, then, defined as $\chi_{d}=\left(\chi_{x}-\chi_{y}\right) / 2$. Figure 1 shows the temperature $(T)$ dependence of the anisotropy of renormalized band $2 \Delta \bar{t} / \bar{t}_{0}$, where $\Delta \bar{t}=\left|\bar{t}_{x}-\bar{t}_{y}\right| / 2=\left|\left(t_{x}-t_{y}\right) \delta / 2+\frac{3}{8}\left(J_{x}-J_{y}\right) \chi_{d}\right|$ and $\bar{t}_{0}=\left(\bar{t}_{x}+\bar{t}_{y}\right) / 2$. The anisotropy is strongly enhanced at lower $T$, especially for lower $\delta$. This enhancement comes from the development of $\chi_{d}$, namely the underlying $d \mathrm{FSD}$ instability in the $t-J$ model. [8, 9, 10] The presence of the anisotropy in $t_{\tau}$ and $J_{\tau}$, however, smears the singularity associated with the $d \mathrm{FSD}$ instability, which would appear below $T \approx 0.03 J(0.05 J)$ for $\delta=0.08(0.07)$ and disappear for $\delta \gtrsim 0.09$ if the input anisotropy is zero.

The irreducible dynamical magnetic susceptibility $\chi_{0}(\mathbf{q}, \omega)$ reads

$$
\chi_{0}(\mathbf{q}, \omega)=\frac{1}{4 N} \sum_{\mathbf{k}} \frac{\tanh \frac{\xi_{\mathbf{k}}}{2 T}-\tanh \frac{\xi_{\mathbf{k}+\mathbf{q}}}{2 T}}{\xi_{\mathbf{k}}-\xi_{\mathbf{k}+\mathbf{q}}+\omega+\mathrm{i} \Gamma},
$$

where $N$ is the total number of (bilayer) lattice sites and $\Gamma$ is a positive infinitesimal. We calculate magnetic excitations in the renormalized random phase approximation (RPA) [30, 31]

$$
\chi(\mathbf{q}, \omega)=\frac{\chi_{0}(\mathbf{q}, \omega)}{1+J(\mathbf{q}) \chi_{0}(\mathbf{q}, \omega)} .
$$

Here

$$
J(\mathbf{q})=2 r\left(J_{x} \cos q_{x}+J_{y} \cos q_{y}\right)+J_{z} \cos q_{z}
$$



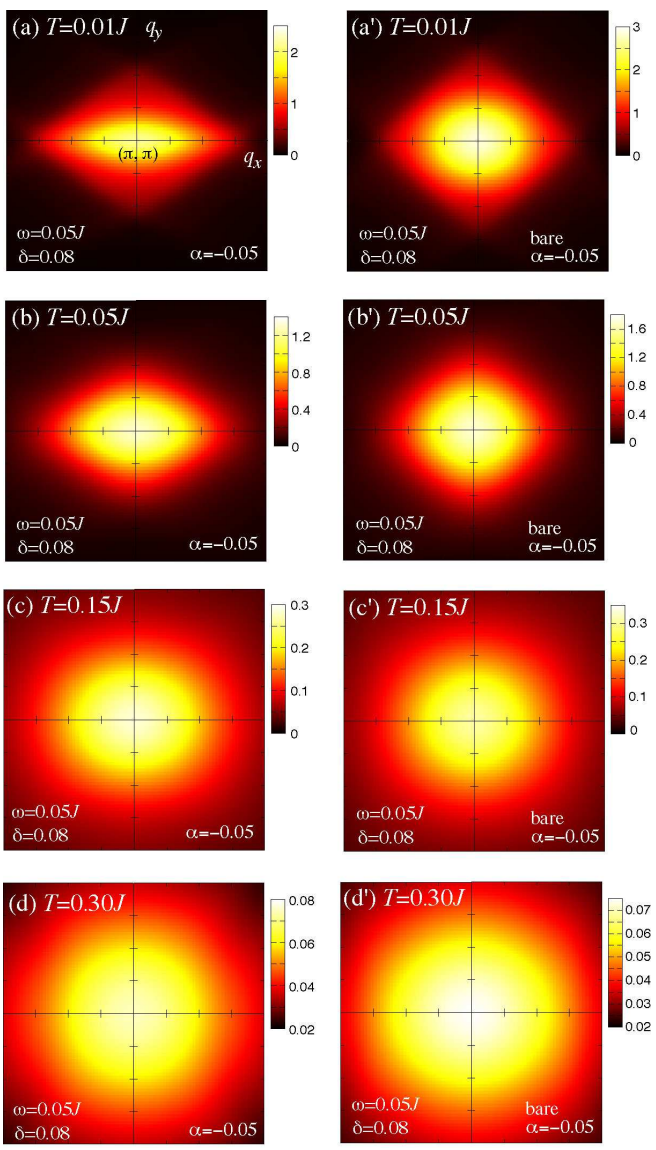

FIG. 2: (Color online) Left-hand panels: q maps of $\operatorname{Im} \chi(\mathbf{q}, \omega)$ for a sequence of $T$ in $0.6 \pi \leq q_{x}, q_{y} \leq$ $1.4 \pi$ for $\delta=0.08, \omega=0.05 \mathrm{~J}$, and $\alpha=-0.05$. Right-hand panels: corresponding results for the bare anisotropy, namely without $d$ FSD correlations.

with a renormalization factor $r$. While $r=1$ in the plain RPA, the value of $r$ is reduced in the renormalized RPA such that the AF instability appears in a doping region comparable with actual materials. We set $r=0.4$, which confines the AF instability to $\delta \lesssim 0.068$.

We calculate $\chi(\mathbf{q}, \omega)$ numerically by choosing $\Gamma=0.05 \mathrm{~J}$. This choice of $\Gamma$ is mainly due to numerical convenience, but simulates damping of electrons by static defects in real materials and broadening due to limited energy resolution in neutron scattering experiments. Since neutron scattering measurements for untwinned YBCO are performed for the odd channel, we focus on this channel $\left(q_{z}=\pi\right)$. Considering that the hole density in $\mathrm{YBCO}_{6.45}$ is estimated as $8.5 \%,[22]$ we fix $\delta=0.08$.

The left-hand panels of Fig. 2 show two-dimensional q maps of the imaginary part of $\chi(\mathbf{q}, \omega)$ at $\omega=0.05 \mathrm{~J}$ for a sequence of temperatures. At $T=0.01 \mathrm{~J}$, significant spectral 


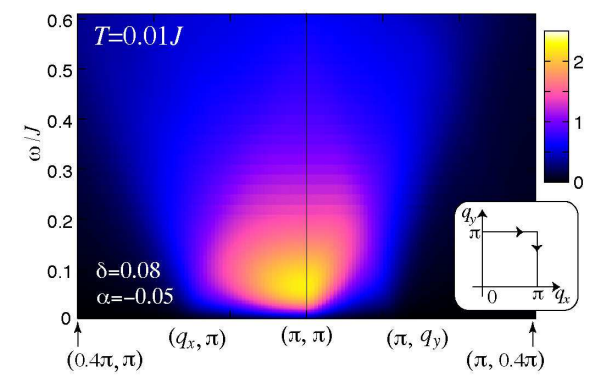

FIG. 3: (Color online) $(\mathbf{q}, \omega)$ map of $\operatorname{Im} \chi(\mathbf{q}, \omega)$ at $T=0.01 J$ for $\delta=0.08$ and $\alpha=-0.05$; the $\mathbf{q}$ directions are shown in the inset.

weight is centered around $\mathbf{q}=(\pi, \pi)$ and is strongly elongated along the $q_{x}$ direction. This pronounced anisotropy originates from the strong enhancement of the original band anisotropy due to $d$ FSD correlations (Fig. 1). The anisotropy of magnetic excitations is reduced with increasing $T$. In particular, the distribution of the spectral weight becomes nearly symmetric around $(\pi, \pi)$ at $T=0.30 J$. To clarify the $d \mathrm{FSD}$ effect, we show the corresponding result for the bare anisotropy effect by switching off $d \mathrm{FSD}$ correlations in the right-hand panels of Fig. 2, where we impose the same anisotropy $\alpha=-0.05$, but assume $\chi_{d} \equiv 0$. Although the value of $\Delta \bar{t}$ does not depend on $T$, we see that the anisotropy of $\operatorname{Im} \chi(\mathbf{q}, \omega)$ depends weakly on $T$ and becomes visible with decreasing $T$. But the obtained anisotropy [Figs. 2(a') and 2(b')] is much weaker than the results due to $d$ FSD correlations [Figs. 2(a) and 2(b)]. Hence the underlying dFSD correlations are particularly important to produce a strongly enhanced anisotropy at lower $T$.

To see the $\omega$ dependence of the anisotropy, the $(\mathbf{q}, \omega)$ map of $\operatorname{Im} \chi(\mathbf{q}, \omega)$ is shown in Fig. 3 at low $T$. While the anisotropic distribution is still discernible for a high energy where the spectral intensity is substantially reduced, a pronounced anisotropy appears especially in a low energy region.

The evolution of the spectral intensity of $\operatorname{Im} \chi(\mathbf{q}, \omega)$ is shown in Fig. 4 as a function of $\omega$ for several choices of $T$ at $\mathbf{q}=\mathbf{Q}=(\pi, \pi)$. At high $T, \operatorname{Im} \chi(\mathbf{Q}, \omega)$ shows a broad maximum at a moderate energy. With decreasing $T$, low-energy spectral weight is substantially enhanced, yielding a peak structure at low energy.

When $d$ FSD correlations strongly enhance a small $x y$ anisotropy of the original band dispersion, a strong anisotropy of $\operatorname{Im} \chi(\mathbf{q}, \omega)$ is induced with a characteristic $T$ dependence. In $\mathrm{YBCO}_{6.45}$, the experimental data[22] suggest that fermions responsible for magnetic exci- 


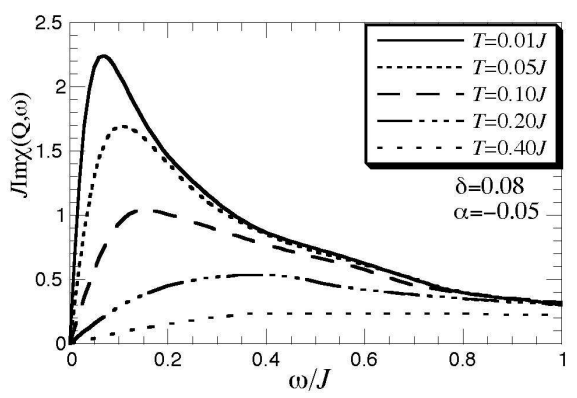

FIG. 4: $\omega$ dependence of $\operatorname{Im} \chi(\mathbf{Q}, \omega)$ for several choices of $T$ for $\delta=0.08$ and $\alpha=-0.05$.

tations feel a rather small mean field of singlet pairing in the sense that neither a gap feature nor a clear impact of the onset of superconductivity is observed in neutron scattering. This special experimental situation is mimicked by setting $\Delta \equiv 0$ in the slave-boson scheme of the $t-J$ model. We have found that this phenomenological treatment well captures the most salient features observed in $\mathrm{YBCO}_{6.45}:[22]$ (i) the strongly enhanced anisotropy of $\operatorname{Im} \chi(\mathbf{q}, \omega)$ at lower $T$ for low $\omega$ (Figs. 2 and 3 ) and (ii) the enhanced spectral weight of $\operatorname{Im} \chi(\mathbf{Q}, \omega)$ at low $\omega$ for low $T$ (Fig. 4).

On the other hand, in the standard slave-boson mena-field theory of the $t$ - $J$ model[18], the onset temperature of singlet pairing is higher than that of the $d F \mathrm{FD}$, and the singlet formation suppresses the susceptibility of the $d$ FSD, which then does not diverge. [8] The susceptibility, however, remains appreciable[17] so that a small external anisotropy introduced in the $t$ - $J$ model is strongly enhanced even in the singlet pairing state. [8] This effect is more pronounced for a lower doping rate. Such an enhanced anisotropy nonetheless turns out not to be sufficient to capture the anisotropy of $\operatorname{Im} \chi(\mathbf{q}, \omega)$ observed in $\mathrm{YBCO}_{6.45}[22$ ] even at qualitative level for a realistic choice of parameters. In particular, the anisotropy is enhanced at relatively high $T$ (Fig. 20 in Ref. 18). Moreover the experimental observation[22] that the onset of superconductivity hardly affects magnetic excitations is difficult to be captured in existing microscopic theories for cuprates.

Inclusion of singlet pairing is not so straightforward, and something beyond the existing theories happens in $\mathrm{YBCO}_{6.45}$. Here focusing on the context of the present paper we discuss several possibilities that $d$ FSD correlations still remain strong enough to be compatible with the present result even in a full calculation. First, since the $d$ FSD instability concerns discrete symmetry breaking, the spontaneous symmetry breaking is allowed at finite temperature even in the exact analysis of the two-dimensional model, in contrast to super- 
conductivity and antiferromagnetism. Hence suppression of the $d \mathrm{FSD}$ due to fluctuations is less crucial than the latter two, in favor of strong correlations of the $d \mathrm{FSD}$. In the presence of a small external $x y$ anisotropy, then, the anisotropy can be strongly enhanced as we have seen in Fig. 1. The feedback of the enhanced anisotropy appears as further reduction of the magnitude of singlet pairing compared with the mean-field value in the slave-boson theory of the $t-J$ model. Second, $\mathrm{YBCO}_{6.45}$ has an orthorhombic crystal structure, but is close to the continuous transition to a tetragonal structure. The $d$ FSD order parameter couples to a phonon related to the orthorhombic-tetragonal structural transition, which contributes to an enhancement of $d$ FSD correlations. In this case, the phonon spectrum may also show strong anisotropy in the orthorhombic phase. Last, there might be a phase segregation into holepoor AF domains with nano-scale correlation lengths and hole-rich superconducting islands in $\mathrm{YBCO}_{6.45}$, and the neutron scattering signals originate mainly from the AF domains. Such finite-sized domains are often discussed in a strongly underdoped superconducting region in $\mu \mathrm{SR}$ measurements. [32, 33]

While the present result well captures the neutron scattering data in $\mathrm{YBCO}_{6.45},[22]$ a detailed comparison reveals disagreement about several aspects, which may be mainly due to our simple calculation ignoring singlet pairing. (i) A weakly incommensurate structure was observed along the $q_{x}$ direction, whereas we have obtained a single peak at $(\pi, \pi)$ as read off from Fig. 2, In a fermiology scenario, the incommensurate structure in Y-based cuprates is explained by the suppression of the commensurate peak due to the development of $d$-wave singlet pairing.[18, 34] Hence the observed weak incommensurate peak can be due to the development of relatively weak singlet pairing. (ii) The observed data at $\omega=50 \mathrm{meV}$ was interpreted to be isotropic.[22] In the present calculation, the anisotropy becomes less pronounced for high $\omega$, but is still discernible (Fig. 3). On the other hand, if we include singlet pairing in the slave-boson mean-field theory, it is known[18] that the anisotropy of $\operatorname{Im} \chi(\mathbf{q}, \omega)$ at high $\omega$ depends strongly on energy. More detailed experiments as well as more complete calculations are necessary to discuss the anisotropy at high $\omega$.

The present phenomenological calculation suggests that $d$-wave singlet pairing is substantially reduced in $\mathrm{YBCO}_{6.45}$, which then concomitantly enhances strong $d \mathrm{FSD}$ correlations, leading to a magnetic excitation spectrum very similar to the experimental data. [22] Since the magnitude of $d$-wave singlet pairing is often discussed to become larger with decreasing doping rate, the present study implies an interesting direction to understand the evolution 
of $d$-wave singlet pairing as well as its connection with $d \mathrm{FSD}$ correlations in underdoped cuprates. Recent angle-resolved photoemission spectroscopy data[35] is in fact suggestive of relatively small $d$-wave singlet pairing. It demonstrated two distinct gaps, the amplitude of one of which increases for lower doping rates while the other, which shows $d$-wave symmetry, decreases. In addition, strong $d$ FSD fluctuations substantially reduce the lifetime of quasiparticles in the antinodal region of the FS while not in the nodal direction as shown in Ref. 36. Hence strong $d \mathrm{FSD}$ fluctuations may play an integral role to understand the pseudogap in the strongly underdoped regime of YBCO. Strong $d$ FSD fluctuations are also expected in a scenario of a quantum phase transition into the $d$ FSD state in a underdoped region. [37] Such a scenario is, however, based on the assumption of the existence of robust $d$-wave pairing, which is different from the present theory where we have invoked the substantial suppression of $d$-wave pairing due to the competition[16, 17] with the $d$ FSD in a strongly underdoped region.

The author is grateful to V. Hinkov and B. Keimer for sharing their unpublished data with him and to W. Metzner and A. Toschi for very helpful discussions.

[1] S. A. Kivelson, I. P. Bindloss, E. Fradkin, V. Oganesyan, J. M. Tranquada, A. Kapitulnik, and C. Howald, Rev. Mod. Phys. 75, 1201 (2003).

[2] J. M. Tranquada, B. J. Sternlieb, J. D. Axe, Y. Nakamura, and S. Uchida, Nature (London) 375, 561 (1995).

[3] T. Niemöller, N. Ichikawa, T. Frello, H. Hünnefeld, N. H. Andersen, S. Uchida, J. R. Schneider, and J. M. Tranquada, Eur. Phys. J. B 12, 509 (1999).

[4] N. Ichikawa, S. Uchida, J. M. Tranquada, T. Niemöller, P. M. Gehring, S.-H. Lee, and J. R. Schneider, Phys. Rev. Lett. 85, 1738 (2000).

[5] M. Fujita, H. Goka, K. Yamada, and M. Matsuda, Phys. Rev. B 66, 184503 (2002).

[6] S. A. Kivelson, E. Fradkin, and V. J. Emery, Nature (London) 393, 550 (1998).

[7] J. Zaanen, Z. Nussinov, and S. I. Mukhin, Ann. Phys. (N.Y.), 310, 181 (2004); V. Cvetkovic, Z. Nussinov, S. Mukhin, and J. Zaanen, Europhys. Lett. 81, 27001 (2008).

[8] H. Yamase and H. Kohno, J. Phys. Soc. Jpn. 69, 332 (2000); 69, 2151 (2000).

[9] A. Miyanaga and H. Yamase, Phys. Rev. B 73, 174513 (2006). 
[10] B. Edegger, V. N. Muthukumar, and C. Gros, Phys. Rev. B 74, 165109 (2006).

[11] C. J. Halboth and W. Metzner, Phys. Rev. Lett. 85, 5162 (2000).

[12] I. Grote, E. Körding, and F. Wegner, J. Low Temp. Phys. 126, 1385 (2002); V. Hankevych, I. Grote, and F. Wegner, Phys. Rev. B 66, 094516 (2002).

[13] C. Honerkamp, M. Salmhofer, and T. M. Rice, Eur. Phys. J. B 27, 127 (2002).

[14] A. P. Kampf and A. A. Katanin, Phys. Rev. B 67, 125104 (2003).

[15] A. Neumayr and W. Metzner, Phys. Rev. B 67, 035112 (2003).

[16] H. Yamase and W. Metzner, Phys. Rev. B 75, 155117 (2007).

[17] H. Yamase, Phys. Rev. Lett. 93, 266404 (2004).

[18] H. Yamase and W. Metzner, Phys. Rev. B 73, 214517 (2006).

[19] C. Stock, W. J. L. Buyers, R. Liang, D. Peets, Z. Tun, D. Bonn, W. N. Hardy, and R. J. Birgeneau, Phys. Rev. B 69, 14502 (2004).

[20] V. Hinkov, S. Pailhès, P. Bourges, Y. Sidis, A. Ivanov, A. Kulakov, C. T. Lin, D. Chen, C. Bernhard, and B. Keimer, Nature (London) 430, 650 (2004).

[21] V. Hinkov, P. Bourges, S. Pailhès, Y. Sidis, A. Ivanov, C. D. Frost, T. G. Perring, C. T. Lin, D. P. Chen, and B. Keimer, Nature Phys. 3, 780 (2007).

[22] V. Hinkov, D. Haug, B. Fauqué, P. Bourges, Y. Sidis, A. Ivanov, C. Bernhard, C. T. Lin, and B. Keimer, Science 319, 597 (2008).

[23] H. F. Fong, P. Bourges, Y. Sidis, L. P. Regnault, J. Bossy, A. Ivanov, D. L. Milius, I. A. Aksay, and B. Keimer, Phys. Rev. B 61, 14773 (2000).

[24] P. Dai, H. A. Mook, R. D. Hunt, and F. Doğan, Phys. Rev. B 63, 54525 (2001).

[25] C. Stock, W. J. L. Buyers, Z. Yamani, C. L. Broholm, J.-H. Chung, Z. Tun, R. Liang, D. Bonn, W. N. Hardy, and R. J. Birgeneau, Phys. Rev. B 73, 100504(R) (2006).

[26] Since the $q_{z}$ modulation of magnetic excitations observed in YBCO is known to be well characterized by a bilayer model, we do not consider a direct coupling to $\mathrm{CuO}$ chains.

[27] Y.-J. Kao and H.-Y. Kee, Phys. Rev. B 72, 24502 (2005).

[28] A. P. Schnyder, D. Manske, C. Mudry, and M. Sigrist, Phys. Rev. B 73, 224523 (2006).

[29] T. Pardini, R. R. P. Singh, A. Katanin, and O. P. Sushkov, Phys. Rev. B 78, 024439 (2008).

[30] J. Brinckmann and P. A. Lee, Phys. Rev. Lett. 82, 2915 (1999).

[31] H. Yamase, H. Kohno, H. Fukuyama, and M. Ogata, J. Phys. Soc. Jpn. 68, 1082 (1999).

[32] Ch. Niedermayer, C. Bernhard, T. Blasius, A. Golnik,A. Moodenbaugh, and J. I. Budnick, 
Phys. Rev. Lett. 80, 3843 (1998).

[33] S. Sanna, G. Allodi, G. Concas, A. D. Hillier, and R. De Renzi, Phys. Rev. Lett. 93, 207001 (2004).

[34] Y. Zha, K. Levin, and Q. Si, Phys. Rev. B 47, 9124 (1993).

[35] K. Tanaka, W. S. Lee, D. H. Lu, A. Fujimori, T. Fujii, Risdiana, I. Terasaki, D. J. Scalapino, T. P. Devereaux, Z. Hussain, and Z.-X. Shen, Science 314, 1910 (2006).

[36] W. Metzner, D. Rohe, and S. Andergassen, Phys. Rev. Lett. 91, 066402 (2003).

[37] E.-A. Kim, M. J. Lawler, P. Oreto, S. Sachdev, E. Fradkin, and S. A. Kivelson, Phys. Rev. B 77, 184514 (2008); Y. Huh and S. Sachdev, Phys. Rev. B 78, 064512 (2008). 